\title{
Monte Carlo-Based Comprehensive Assessment of PV Hosting Capacity and Energy Storage Impact in Realistic Finnish Low-Voltage Networks
}

\author{
Ammar Arshad * DD, Verner Püvi and Matti Lehtonen \\ Department of Electrical Engineering and Automation, Aalto University, Maarintie 8, 02150 Espoo, Finland; \\ verner.puvi@aalto.fi (V.P.); matti.lehtonen@aalto.fi (M.L.) \\ * Correspondence: ammar.arshad@aalto.fi; Tel.: +35-846-967-9949
}

Received: 8 May 2018; Accepted: 4 June 2018; Published: 6 June 2018

\begin{abstract}
The direction taken towards sustainable power system and renewable energy generation is now irreversible. The power grid needs to host more renewable energy sources, such as solar power, and tackle power quality problems that come along with it. In this paper, firstly, the Hosting Capacities (HCs), of Photo-Voltaic (PV), were found for various regions and their limiting constraints were defined. Afterwards, comparison was made with the HC values obtained for different voltage value standards defined by various countries. Next, single-phase PV connection percentages in the network were defined that makes the voltage unbalance the limiting factor for HC. Lastly, the HC of the solar generation coupled with a Battery Energy Storage System (BESS) was assessed for the Finnish Low-Voltage (LV) grids. Different BESS-based scenarios were employed and their impact on voltage unbalance and HCs were observed. Finally, also the load voltage unbalance was incorporated to make the approach realistic and its impact on HC was analyzed. Results reveal that, depending on the connection strategy, the BESS can increase as well as decrease the HC based on voltage unbalance criteria. However, the load voltage unbalance has little effect on the solar HC values.
\end{abstract}

Keywords: Photo-voltaic; Low-Voltage networks; Monte-Carlo simulations; battery energy storage system; hosting capacity

\section{Introduction}

Increasing penetration of Photo-Voltaic (PV) in the distribution system, throughout Europe, requires a complete review of the technical constraints of the network. Mainly, the constraints are dependent on the type of PV system connection at the customer vicinity. It can be three-phase or single-phase dependent on the capacity of the PV system and the point of integration. In Europe, the distribution of PVs among Low-Voltage (LV) networks made up $70 \%$ of the total integrated capacity of the power system [1]. The introduction of PVs in the LV networks has reformed the normal power flow scenario and it occasionally flows upstream, during high renewable generation periods.

Most utilities tend to have a general review of network constraints occasionally, to keep themselves up to date with the changing requirements of the network. Voltage rise is a common problem associated with PV integration in LV networks [2]. Moreover, voltage imbalance arises from the random phase connections of the single-phase PVs [3] that are very common in LV networks. Transformer and cable sizing also changes with the load demand and distributed generation capacity. Maximum PV capacity integration in the LV network, without the network's constraints violation, is termed as Hosting Capacity (HC). HC studies quantify the bottleneck of distributed generation integration compared with the network constraints. 
Various studies have been conducted for determining the HCs of distribution networks, utilizing various performance indices as the deciding criteria. In the studies conducted in $[4,5]$, voltage violation criterion was considered as the HC defining index. In these studies, PVs were attached at the beginning and the end of the network that is not realistic. Moreover, the conductor's thermal limits and transformer capacity were the HC limiter in [6,7], respectively. In [8], voltage unbalance is the constraint utilized for HC definition. Versatilities of size and location of PV are modelled by conducting Monte Carlo (MC)-based studies in [9-11]. In [9], HC is measured utilizing the multi-scenario approach, for instance variable positioning of the PVs along the feeder, different power factor of the inverters etc. In addition to the various performance indices, different geographic locations and scenarios were considered, for instance rural, intermediate and urban regions, in [10] and [11]. Moreover, in [11], a 100\% single-phase scenario of PV connection is depicted by making connections to only one phase rather than randomly dividing it among the three phases. It simulates the worst-case scenario but is different from the normal practices of PV integration. Thus, an approach should be deigned that replicates the realistic model of PV integration in the LV network.

The stochastic framework is a comprehensive way to evaluate the hosting capacity of the PVs. The MC-based models are advantageous over deterministic calculations due to their faster calculation speed. Many MC runs can be simulated faster than all possible combinations of input variables in a deterministic approach. In fact, the random values of the input variables can be utilized in MC simulations, which fits some values that are probabilistic in their nature (e.g., solar irradiance). However, the probability distributions of the input variables should be carefully chosen in order to represent the real life cases. Finally, the results of MC simulations can be analyzed by means of statistical methods and HC value can be found with respect to the risk level that the grid is willing to tolerate.

In literature, a comprehensive analysis was conducted in [12] where several influence factors were considered such as background voltage unbalance, solar inverter penetration level and inverter size affecting the voltage unbalance. The results of stochastic voltage unbalance assessment were compared with the deterministic voltage unbalance calculation and the results of stochastic assessment demonstrated bigger flexibility in results analysis. It was shown that connecting a solar inverter to the phase with lowest phase voltage can have good impact on the voltage unbalance mitigation. However, having Battery Energy Storage System (BESS) and load voltage unbalance can change the voltage magnitudes over time and, eventually, PV would need to be constantly switched to the phase with lowest voltage level.

The BESS has a significant influence on distribution grid operation. It participates in demand response and affects the power quality in the grid. While saving the excess energy during high generation hours, it provides the energy for the grid during high demand. The BESS is an important part of the grid of the future with high renewable energy penetration levels and will be more commonly installed in households, as the price of the batteries decreases gradually.

Various studies have been conducted assessing the impact of the batteries on the voltage unbalance in [13-15]. In [13], the single-phase BESS was connected to a grid with single-phase solar and wind generators. The voltage unbalance, caused by the load, was also considered and the voltage unbalance resulting from several sources was analyzed. The study shows that the BESS can reduce voltage unbalance levels by shaving the demand peaks; however, it strongly depends on the phase in which the BESS is connected. In [14], a battery-power-flow control algorithm is employed with BESS inverter that injects real and reactive power to the grid in order to mitigate the voltage unbalance and network losses. It was demonstrated that having BESS in the same phase as PV can reduce the voltage unbalance significantly. In [15] two more BESS were added to the grid-BESS for every phase. The fuzzy controller was then utilized to control the three BESS to mitigate the voltage unbalance and keep voltage levels within acceptable limits.

It can be concluded that single-phase BESS has bigger impact on power quality parameters rather than three-phase BESS. The single-phase BESS, as well as PV, injects the imbalanced current to a grid causing the voltage unbalance and sets restrictions on the penetration level. On the other hand, it is 
cheaper and easier to install in a household due to its smaller size. The three-phase BESSs are more expensive but allow connecting higher number of PVs to a grid without disturbing the current balance in the three-phase system.

The study presented in this paper simulates the more realistic approach to randomly distributed PVs, among the nodes and phases of the network, to evaluate the HCs and limiting constraints of various scenarios. The main idea of this study is to assess HC of PVs in a Finnish distribution grid with respect to added BESS and load voltage unbalance. The study is relevant to other North European countries with sparsely populated areas. Moreover, it compares the MC simulations-based HC values of the LV networks (formulated in [11]), with different voltage limits as described by various countries with the standard EN 50160 [16] i.e., $\pm 10 \%$ voltage change from the nominal. The considered voltage bands are $\pm 5 \%$ and $-5 /+3 \%$ (German standard [17]). Furthermore, the transformer capacity is taken as a reference for measuring the HCs of the networks.

Firstly, the HC of the various LV networks were defined based on the limiting constraints on each scenario. The PV attachment scenarios considered were balanced (three-phase) and unbalanced (single-phase). Once the limiting constraints were defined, the scenarios in which the voltage unbalance was the limiting factor (in the unbalanced PV case), were further investigated to quantify the percentage of balanced and unbalanced PVs were not violating the voltage unbalance limit i.e., $2 \%$ of negative sequence voltage. Secondly, the battery is employed with the PV systems for the maximal use of generation by self-consumption of the customer and its effect on HC was studied. Only the single-phase PV owners were assumed to have BESS installed. Furthermore, several scenarios were investigated, such as:

1. $\quad$ PV and BESS on random phases

2. PV and BESS on same phase

3. Addition of load unbalance to the scenario 2

The effect of each scenario on the voltage unbalance criteria and network's HC was then measured. In case 3, load unbalance was incorporated to make the simulations more realistic. The load unbalance value used was based on measured voltage unbalance data of a single household in Finland.

\section{Source Data}

\subsection{Test Networks and Limiting Constraints}

For the determination of HC, this study utilizes Finnish LV networks, formulated in [11]. Networks were constructed while considering voltage drop and current loading as the deciding factors, as they signify the length of the network. Moreover, transformer capacity defined the number of feeders considered for each type of network. Three Medium Voltage (MV) change scenarios $(0 \%, \pm 5 \%)$ were considered and for each case different networks were developed that fully utilize the network limits without violating the constraints. The constraints of the network considered were:

1. Load flow convergence violation (E1)

2. Under-voltage (E2)

3. Over-voltage (E3)

4. Negative-Sequence-Voltage unbalance (E4)

5. Cable ampacity violation (E5)

6. Neutral-wire ampacity violation (E6)

7. Transformer capacity exceeded (E7)

In the defined constraints, voltage violations (E2, E3) are related to the standard EN50160 i.e., $U_{n} \pm 10 \%$ and the ampacity violations are based on the current ratings of the cables.

Moreover, customer's data utilized was of three different types, divided based on heating modes and the percentage division is presented in Table A1. The regions are Predominantly Rural (PR), 
Intermediate (IN) and Predominantly Urban (PU). Moreover, LV grid parameters utilized are presented in Table A2. The formulated networks and their limiting constraints that will be utilized for further investigation of HC are defined in Table 1.

Table 1. Test networks for different regions and Medium Voltage (MV) change scenarios.

\begin{tabular}{ccccc}
\hline Region & MV Change & No. of Feeder & Nodes per Feeder & Total Customers \\
\hline \multirow{3}{*}{ PR } & $-5 \%$ & 1 & 6 & 6 \\
& $0 \%$ & 1 & 8 & 8 \\
& $5 \%$ & 1 & 10 & 10 \\
\hline \multirow{3}{*}{ IN } & $-5 \%$ & 3 & $3,2,2$ & 28 \\
& $0 \%$ & 3 & $4,3,3$ & 40 \\
& $5 \%$ & 3 & $5,5,4$ & 56 \\
\hline \multirow{2}{*}{ PU } & $-5 \%$ & 3 & $2,1,1$ & 240 \\
& $0 \%$ & 3 & $2,2,1$ & 300 \\
& $5 \%$ & 3 & $2,2,1$ & 300 \\
\hline
\end{tabular}

\subsection{Solar and BESS Power Variation Data}

The solar power data used in this research is based on [18]. A one-year solar power curve was modelled of a solar array located in Helsinki area, Finland. Panels are tilted at $45^{\circ}$ and are oriented to the south. To define the maximum PV hosting capacity, the PV generation pattern follows the theoretical maximum of solar generation, neglecting the possible weather conditions hindering solar irradiation. The BESS is assumed as single-phase home battery, Tesla Powerwall 2 that has robust environmental specifications, which makes it a viable option for a household. The power curves are found by subtracting solar power generated and load demand at the same node.

\subsection{Load Unbalance Data}

Load voltage unbalance data utilized is measured on a single household in Finland. The measured data is processed to get a lognormal distribution function. Later, the magnitude of the voltage unbalance is sampled from the distribution function and the angle of the unbalance is sampled between phases $a, b$ and $c$ with uniform probability. The mean and standard deviation of the corresponding normal distribution are $\mu=-1.33$ and $\sigma=0.39$. The probability density function and the cumulative distribution function of the load voltage unbalance is depicted in Figure 1.

\section{Analysis Methodology for PV Hosting Capacity Determination}

\subsection{Worst Case Hours for PV-Only Strategy}

In this paper, PV hosting capacity is defined as the ratio of total PV generation of the LV network to the respective feeding transformer capacity. In [11], maximum network load was used for scaling $\mathrm{HC}$ rather than transformer capacity. In that case, $\mathrm{HC}$ varies with the varying peak load of the network. As the network nodes differ based on MV change value $( \pm 5 \%, 0 \%)$, HC values calculated this way will be dependent on the network nodes, in turn on its peak load. However, if transformer capacity is taken as a reference all the HCs for a particular region are easily comparable. For HC determination, MC simulations are based on worst-case hours, instead of all the 8760 hourly data sets. The data sets having large PV generation and minimum loading, located in the top left corner of the convex hull, are considered, as shown in Figure 2. 


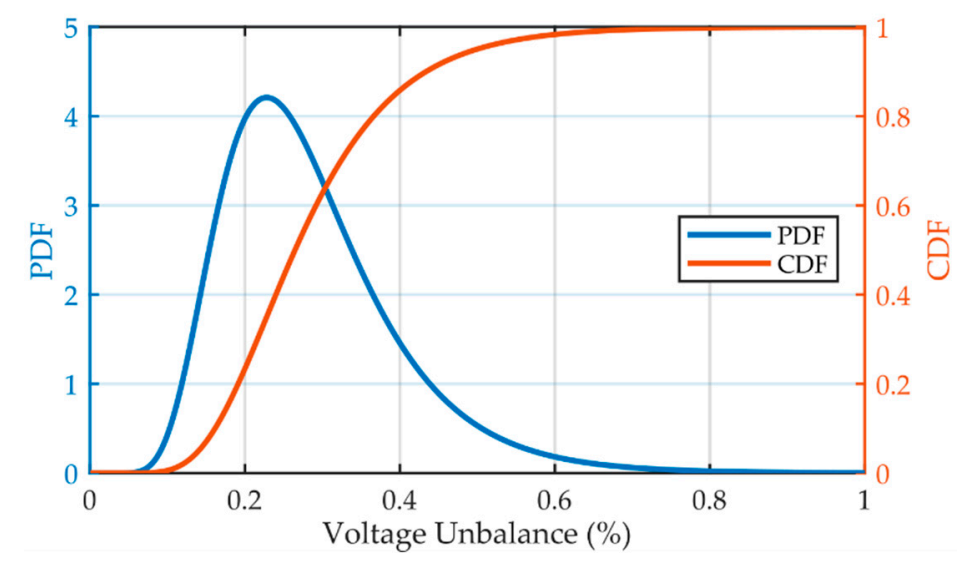

Figure 1. Probability Density Function (PDF) and Cumulative Distribution Function (CDF) of load voltage unbalance.

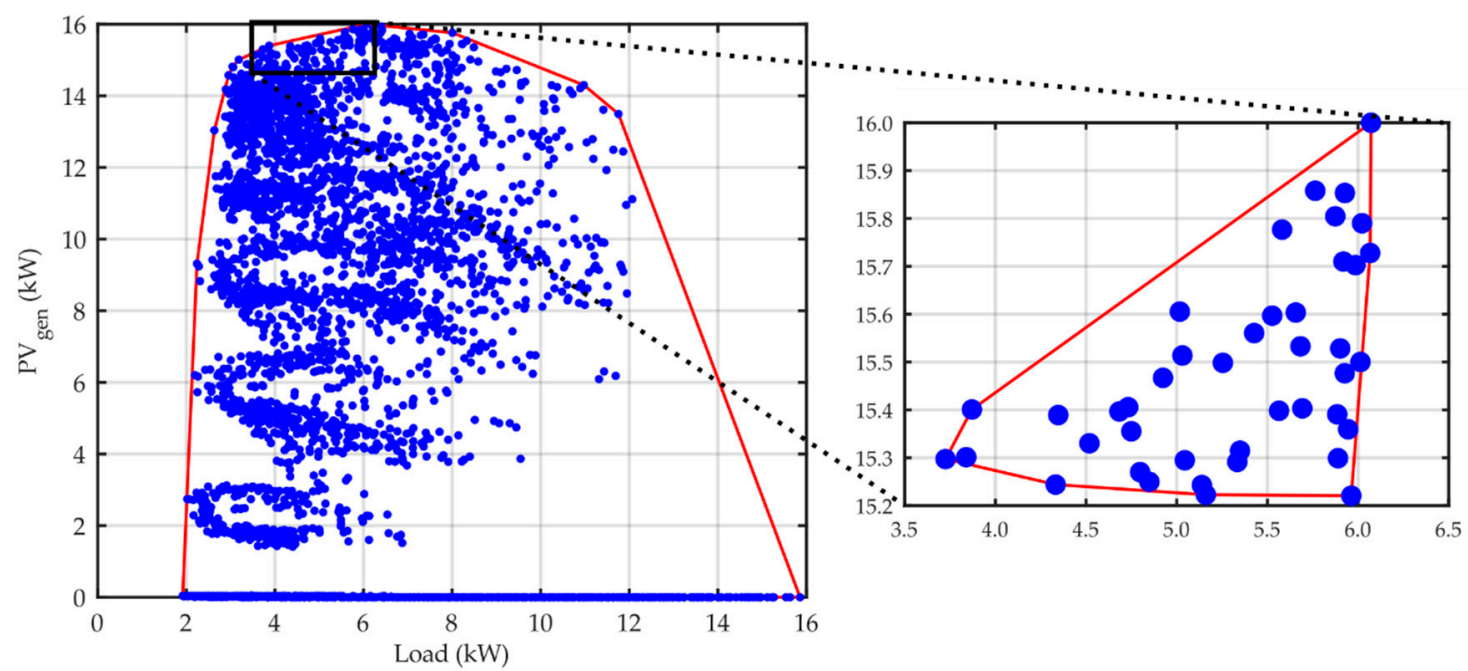

Figure 2. Plot for computing worst-case hour for Hosting Capacity (HC) simulations (Photo-Voltaic (PV)-only strategy) in Predominantly Rural (PR) region.

\subsection{Worst Case Hours with BESS}

Predominantly, residential areas may have single-phase PV connections. As the single-phase connections are the primary reason for the voltage unbalance introduction, different BESS connection strategies can be utilized to achieve higher HCs in case of higher unbalanced PV penetrations.

In cases with BESS connected to the grid, the worst-case hours are chosen from the region as shown in Figure 3. A graph is plotted between the maximum PV generation of the network and battery capacity for all hours of a whole year. BESS power is positive when PV generation is bigger than load and charging. On the contrary, BESS power is negative while it is feeding the load. The maximum power of BESS is limited though by the rated power shown in the data sheet [19]. To show the biggest possible impact of the BESS at every possible instant, the storage capacity is neglected. The top right corner of the plot represents the worst case hours for the BESS connected scenario, as the power flows of PV and BESS are the biggest. Both of the devices are active simultaneously and represent full contribution to the HC. The bottom-left corner of the plot is neglected due to the fact that these hours represent dark time of a year during winter, when PV generation is at zero and high household heating demand is present. 


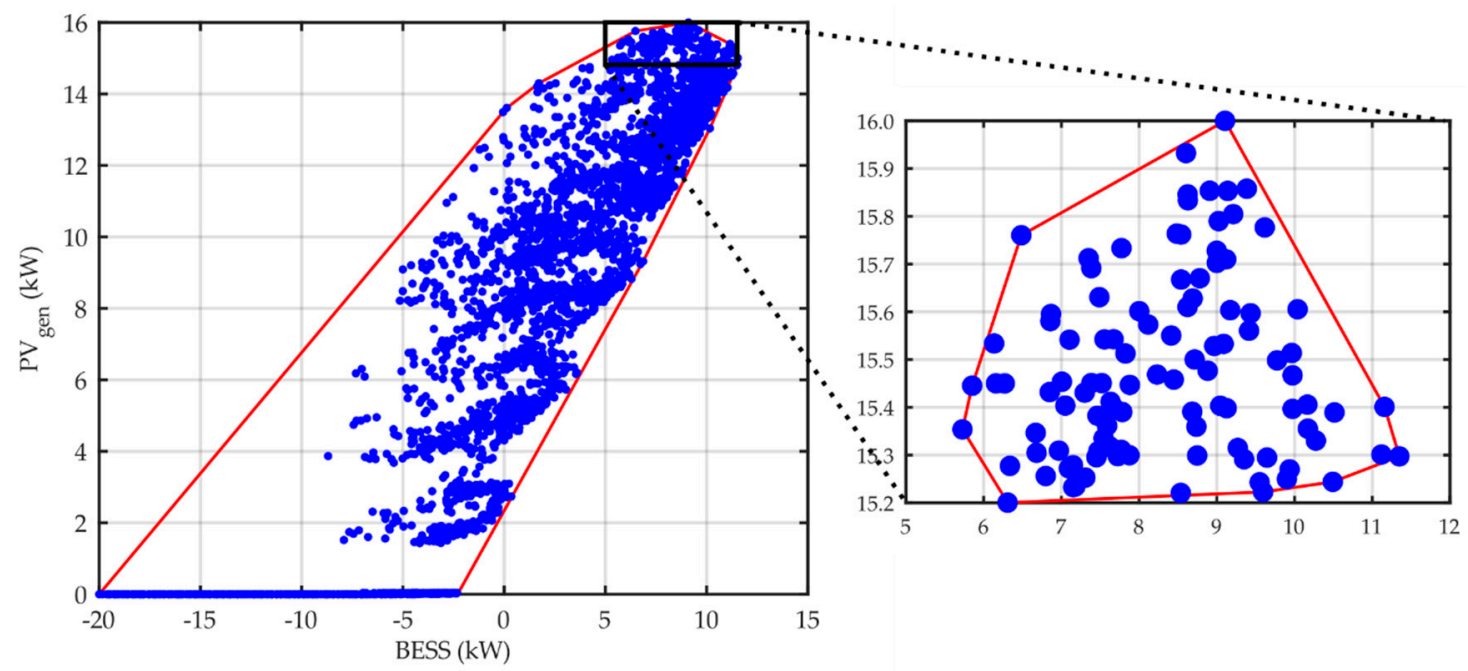

Figure 3. Plot for computing worst-case hour for HC simulations (PV and Battery Energy Storage System (BESS) strategies) in PR region.

\subsection{Monte-Carlo Based Algorithm}

Figure 4 depicts, different stages involved in the MC-based HC determination algorithm. Stage 1 defines the network parameters based on the type of network chosen. Once the specific network is determined, stage 2 commences with the definition of loading scenario and worst case data sets. Loading scenarios are based on the probabilities of different types of customers based on the region. As the region changes, the load probability of households varies and the loading profile of the whole network changes. Thus, worst-case hours differ from one region to another, as they are dependent on the loading scenarios. There are 1000 iterations of stage 2 and in each iteration, different loading scenarios are considered dependent on the regions' loading probability that in turn modifies the worst-case hour datasets. Once the small set of worst-case hours is selected, stage 3 iterates 10 times while randomly selecting values from the worst-case hours. Lastly, load flow is computed for the predefined network, and the constraints violations are determined. PV size is incremented in every stage 1 iteration, until the violations exceed $2.5 \%$ of the total number of iterations. 


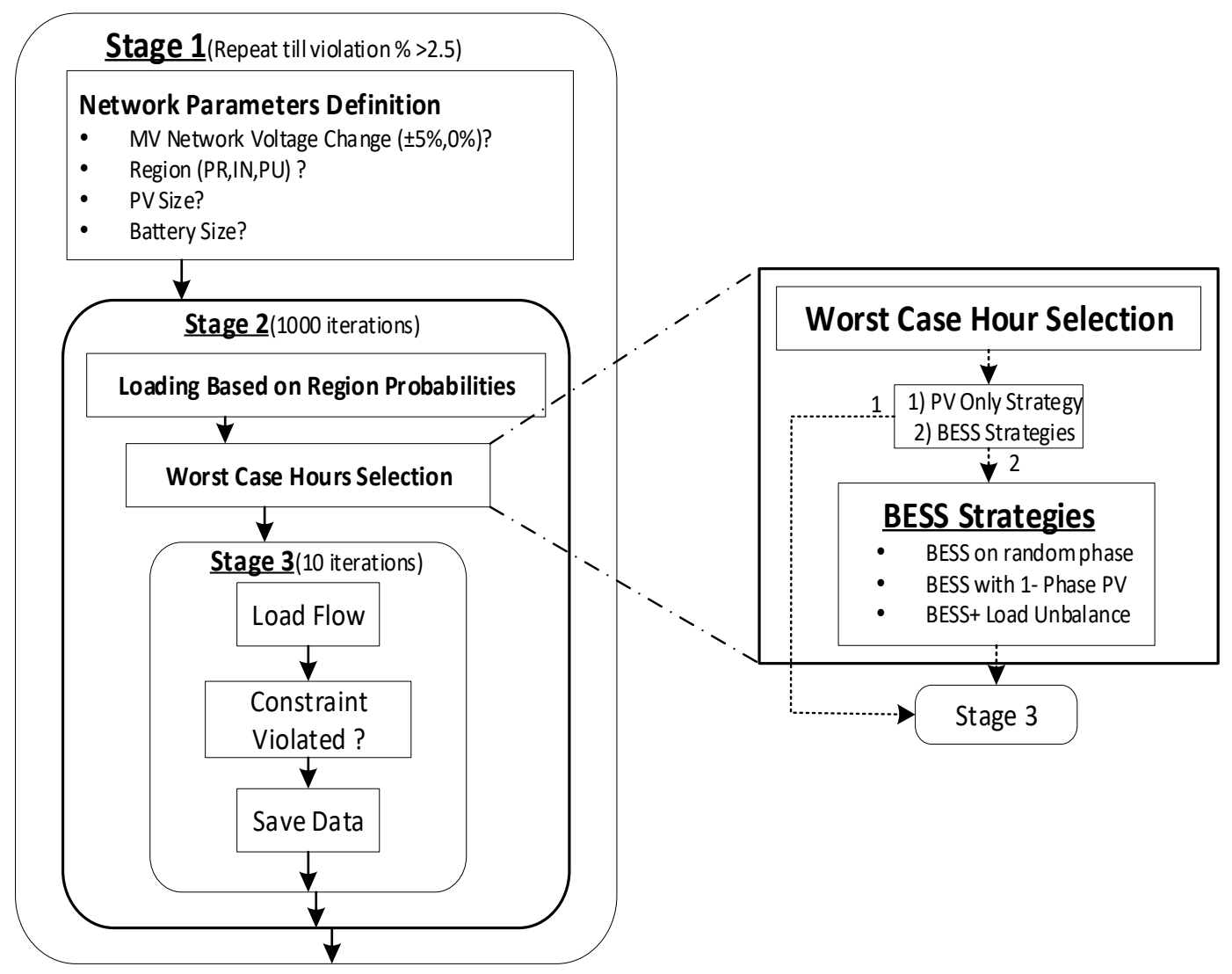

Figure 4. Monte Carlo (MC)-based algorithm for HC determination. Predominantly Rural (PR), Intermediate (IN) and Predominantly Urban (PU).

\section{Case Studies and Results}

This section evaluates the limiting constraints for Finnish LV networks. HC is computed for all the grids based on the geographically distinct regions. The mean value of $\mathrm{HC}$ capacity corresponding to the $2.5 \%$ limit violation probability and its limitations are computed for:

1. PV-only cases
a. $\quad 100 \%$ Balance PVs (Three-Phase Connections) case
b. $\quad 100 \%$ Unbalanced PVs (Single-Phase Connections) case
c. Varying Percentages of Single and Three-phase PV connections

2. BESS connected cases
a. Random BESS connection
b. BESS on same phase as Single-Phase PV
c. Load voltage unbalance added to BESS strategy $b$

In all the cases, limiting constraints will be the same as discussed in Section 2, except the voltage violation limits. The limits of E2 and E3 will be modified based on different country-based standards for comparison case study.

\subsection{Base Case: $\Delta U= \pm 10 \%($ Standard EN 50160)}

Two different sets of MC simulations are conducted for the base case in which voltage violation band is according to standard EN 50160 i.e., $\pm 10 \%$ of the nominal voltage. For balanced feed in case, 
transformer loading is the main issue in most of the scenarios. In PR region, $0 \%$ and $5 \% \mathrm{MV}$ change simulations, over voltage (E3) is the limiting factor, while in PU region; cable ampacity (E5) is limiting the maximum HC capability of the $-5 \%$ MV change case network. However, in the unbalance feed in case, PR and IN region networks has negative sequence voltage unbalance (E4) as the limiting constraint. In PU region, neutral-wire ampacity (E6) is the primary issue and E4 is the secondary concern. Furthermore, largest HC reduction is observed in the PR region in case of unbalanced PV connections. All the limiting constraints and mean HCs of different cases under consideration are presented in Table 2.

Table 2. Test Networks' mean Hosting Capacities (HCs) and their limiting constraints.

\begin{tabular}{cccccc}
\hline & \multicolumn{2}{c}{ Balanced (3-Phase) } & \multicolumn{2}{c}{ Unbalanced (1-Phase) } \\
\hline \multirow{2}{*}{ Region } & MV Drop & Limiting Constraint & $\mathbf{u}_{H C}(\%)$ & Limiting Constraint & $\mathbf{u}_{\text {HC }}(\%)$ \\
\hline \multirow{3}{*}{ PR } & $-5 \%$ & E7 & 110.3033 & E4 & 23.2201 \\
& $0 \%$ & E3 & 105.2661 & E4 & 24.7720 \\
& $5 \%$ & E3 & 50.3049 & E4,E3 & 25.1611 \\
\hline \multirow{3}{*}{ IN } & $-5 \%$ & E7 & 105.6751 & E4 & 52.8377 \\
& $0 \%$ & E7 & 110.3156 & E4 & 58.0569 \\
& $5 \%$ & E7 & 113.7825 & E4 & 65.0218 \\
\hline \multirow{3}{*}{ PU } & $-5 \%$ & E5 & 93.2688 & E6,E4 & 30.3116 \\
& $0 \%$ & E7 & 107.8319 & E6,E4 & 40.7981 \\
& $5 \%$ & E7 & 107.7761 & E6,E4 & 40.8671 \\
\hline
\end{tabular}

\subsection{Case II: Base Case Comparison with $\Delta U= \pm 5 \%$ and $\Delta U=-5 /+3 \%$ (German LV Standard) Cases}

All the constraints defined in Section 2 remain more or less the same for different countries' standards, except the voltage violation band. Therefore, a new set of simulations was conducted for varying voltage violation bands and its effects on the limiting constraints and mean HCs for different cases were observed. Moreover, only the $0 \% \mathrm{MV}$ case is simulated, as LV transformer, in most of the cases, is not equipped with the on-load tap changers. The simulation results show that the HCs of IN and PU region are not affected by the change in the voltage violation band as they are limited by the transformer capacity (E7) in balanced PV input scenarios.

The changes in the HCs and the limiting constraints are observed only in the PR region, where the over voltage is the limiting factor for all the voltage bands employed in the balanced feed in case. The HCs are also reduced significantly, in balanced feed in case of PR region; it is reduced to half in $\pm 5 \% \Delta \mathrm{U}$ case and to one third in $-5 /+3 \% \Delta \mathrm{U}$ case. Furthermore, in single-phase scenarios, HCs do not change much in different regions, but the over-voltage is the primary concern in German LV standard constraints for PR and PU regions. While, for IN region voltage unbalance remains the primary limiting constraint. The observed results are presented in Table 3.

Table 3. Test Networks' mean HCs and their limiting constraints with different voltage bands.

\begin{tabular}{|c|c|c|c|c|c|c|}
\hline \multirow[b]{2}{*}{ Region } & \multirow[b]{2}{*}{ MV Drop } & \multirow[b]{2}{*}{$\Delta \mathbf{U}$} & \multicolumn{2}{|c|}{ Balanced (3-Phase) } & \multicolumn{2}{|c|}{ Unbalanced (1-Phase) } \\
\hline & & & Limiting Constraint & $\mathbf{u}_{H C}(\%)$ & Limiting Constraint & $\mathbf{u}_{H C}(\%)$ \\
\hline \multirow{3}{*}{ PR } & \multirow{3}{*}{$0 \%$} & $\pm 10 \%$ & E3 & 105.2661 & $\mathrm{E} 4$ & 24.7721 \\
\hline & & $\pm 5 \%$ & E3 & 54.1924 & E3-E4 (both) & 24.7798 \\
\hline & & $-5 /+3 \%$ & E3 & 35.6077 & E3 & 17.0346 \\
\hline \multirow{3}{*}{ IN } & \multirow{3}{*}{$0 \%$} & $\pm 10 \%$ & E7 & 110.3156 & E4 & 58.0569 \\
\hline & & $\pm 5 \%$ & E7 & 110.3075 & E4 & 58.0591 \\
\hline & & $-5 /+3 \%$ & E7 & 110.3255 & E4, E3 & 58.0564 \\
\hline \multirow{3}{*}{ PU } & \multirow{3}{*}{$0 \%$} & $\pm 10 \%$ & E7 & 107.8319 & E6, E4 & 40.7981 \\
\hline & & $\pm 5 \%$ & E7 & 107.8106 & E6, E4 & 40.7950 \\
\hline & & $-5 /+3 \%$ & E7 & 107.8090 & E3 & 37.8780 \\
\hline
\end{tabular}




\subsection{Case III: Single-Phase PV Percentage for Voltage Unbalance as a Limitation}

Section 4.2 presented the limiting constraints for the grids when $100 \%$ of the PVs are either single-phase or three-phase, in respective unbalanced and balanced PV connection scenarios. To calculate the percentage of single-phase PVs that does not yet violate the $\mathrm{E} 4$ constraint, a new set of simulations are run that increase the percentage of single-phase PVs in steps until the E4 constraint is violated. Table 4 presents the changing HCs of different regions and their changing constraints with the introduction of unbalanced PVs in the network. The results observed showed that in $0 \%$ MV Drop, in PR region, 10\% single-phase PVs will shift the limiting constraint from over-voltage to voltage unbalance and for IN region $25 \%$ unbalanced PV will cause the voltage unbalance in the system. The PU region has the E6 as the primary limiting constraint, so that region is not included in this study. The results for the +5 and $-5 \%$ MV changes scenarios with the varying unbalanced PV connections are presented in Table 4. The Normal trend depicts that as the single-phase PV are increasing in the network HC is decreasing and the limiting constraint is shifting towards E4.

Table 4. Test Networks' mean HCs and limiting constraints with varying unbalanced PV percentage.

\begin{tabular}{|c|c|c|c|c|c|}
\hline Region & MV Drop & Unbalanced (1-Phase) & Balanced (3-Phase) & Limiting Constraint & $\mathbf{u}_{H C}(\%)$ \\
\hline \multirow{6}{*}{ PR } & \multirow[b]{2}{*}{$-5 \%$} & 0 & 100 & E7 & 110.3033 \\
\hline & & 5 & 95 & E4 & 80.1243 \\
\hline & \multirow[b]{2}{*}{$0 \%$} & 5 & 95 & E3, E4 & 77.4206 \\
\hline & & 10 & 90 & E4 & 63.479 \\
\hline & \multirow{2}{*}{$+5 \%$} & 80 & 20 & $\mathrm{E} 3, \mathrm{E} 4$ & 27.0922 \\
\hline & & 90 & 10 & E4, E3 & 25.1612 \\
\hline \multirow{6}{*}{ IN } & \multirow[b]{2}{*}{$-5 \%$} & 10 & 90 & E7 & 107.0208 \\
\hline & & 15 & 85 & E4 & 90.7603 \\
\hline & \multirow{2}{*}{$0 \%$} & 20 & 80 & $\mathrm{E} 7, \mathrm{E} 4$ & 110.3139 \\
\hline & & 25 & 75 & E4 & 102.5794 \\
\hline & \multirow{2}{*}{$+5 \%$} & 25 & 75 & $\mathrm{E} 7, \mathrm{E} 4$ & 116.4957 \\
\hline & & 30 & 70 & E4 & 108.3732 \\
\hline
\end{tabular}

\subsection{Case IV: Different BESS Connection Strategies}

This section simulation analyzed the effects of BESS introduction in the network on case respective HCs. BESS can have big impact on the HC due to the peak shaving of the PV generation. On the other hand, solar HC can reduce significantly due to the imbalanced current of the BESS being injected to the grid. Simulation results of $0 \% \mathrm{MV}$ drop scenario, for all the different regions and cases, are presented in Table 5.

Table 5. Test Networks' mean HCs and limiting constraints for all cases and regions for 0\% MV drop.

\begin{tabular}{|c|c|c|c|c|c|c|}
\hline Region & Case & MV Drop & Unbalanced (1-Phase) & Balanced (3-Phase) & Limiting Constraint & $\mathbf{u}_{H C}(\%)$ \\
\hline \multirow{6}{*}{ PR } & \multirow[b]{2}{*}{ A } & \multirow{2}{*}{$0 \%$} & 0 & 100 & E7 & 105.2779 \\
\hline & & & 5 & 95 & E4 & 23.2247 \\
\hline & \multirow{2}{*}{ B } & \multirow{2}{*}{$0 \%$} & 50 & 50 & E3, E4 & 114.5631 \\
\hline & & & 60 & 40 & $\mathrm{E} 4$ & 111.4671 \\
\hline & \multirow{2}{*}{$\mathrm{C}$} & \multirow{2}{*}{$0 \%$} & 30 & 70 & E3, E4 & 109.9174 \\
\hline & & & 50 & 50 & E4, E3 & 111.4567 \\
\hline \multirow{6}{*}{ IN } & \multirow{2}{*}{ A } & \multirow{2}{*}{$0 \%$} & 0 & 100 & E7 & 110.3371 \\
\hline & & & 10 & 90 & $\mathrm{E} 4$ & 81.2517 \\
\hline & \multirow{2}{*}{ B } & \multirow{2}{*}{$0 \%$} & 70 & 30 & E7 & 158.6821 \\
\hline & & & 80 & 20 & E4, E7 & 170.3027 \\
\hline & \multirow[b]{2}{*}{$\mathrm{C}$} & \multirow{2}{*}{$0 \%$} & 70 & 30 & E7 & 158.7524 \\
\hline & & & 80 & 20 & E4, E7 & 164.4896 \\
\hline
\end{tabular}




\subsubsection{Case A: BESS on Random Phase}

In this scenario, BESS is attached randomly among phases and there is no coordination between the PV and BESS attachment to the phase. Every phase has equal probability to be chosen for BESS as well as for PV. However, only those nodes are considered for BESS attachment, which have a single-phase PV attached. The results showed that HC falls drastically by having PV and BESS at random phases. In the PR region, the $\mathrm{HC}$ is around $23 \%$, having almost triple fall at 5/95 single/three-phase PV ratio. In the IN region, the $\mathrm{HC}$ falls from $110 \%$ to $81 \%$ at $10 / 90 \mathrm{PV}$ ratio. The E4 becomes the primary limiting constraint of $\mathrm{HC}$ as the voltage unbalance increases with the random BESS connections.

\subsubsection{Case B: BESS on Same Phase as Single-Phase PV}

In case B, the BESS is connected to the same phase as the single-phase PV. In this strategy, the excessive PV generation will be absorbed by the BESS through the same phase wire, reducing the imbalanced current flow in the nodes of the grid. The simulation results show the biggest potential in increasing the solar HC. Due to the voltage unbalance decrease in the PR region, the share of single-phase PVs has risen from 5\% to 50\% without violating E4 limit. In the IN region, the voltage unbalance level has fallen to the levels, where 70/30 single/three-phase PV share is possible without violating E4 constraint.

\subsubsection{Case C: Load Unbalance Consideration}

In case $C$, the voltage unbalance caused by the load is added to the calculated voltage unbalance due to single-phase PV connections. This case represents the realistic LV network scenario, where the loads are connected to different phases, causing unbalanced current flows. In this case, the BESSs are assumed to be connected to the same phase with PV as in Case B. The results reveal that the voltage unbalance applies bigger hindrance to the HC levels; HCs are slightly decreased compared with case $\mathrm{B}$. In PR region, the $\mathrm{HC}$ due to the $\mathrm{E} 4$ has fallen from 50/50 to 30/70 single/three-phase PV ratio. The HC value has fallen slightly as well from $115 \%$ to $111 \%$ at $50 / 50$ PV ratio. In the IN region, the PV ratio remains the same. However, the HC value falls from $170 \%$ to $164 \%$ at $80 / 20 \mathrm{PV}$ ratio.

\section{Discussion}

\subsection{Monte-Carlo Simulations}

The number of iterations considered, for each stage, defines the success probability of MC-based methodology. The small number of stage 2 iterations will lead to the high variation in the HC values yielded by the proposed method. Moreover, the large number of iterations will consume excessive computational time to converge to a solution that can be obtained with fewer iterations. For instance, various simulations utilizing 100 iterations for second stage have standard deviation value of 0.9039 . However, for 500 and 1000 iterations variance in the computed values are 0.8815 and 0.0139 , respectively (results are for PR region at $0 \%$ MV change scenario and utilizing case C). Thus, these small deviations from the mean result will lead to the wrong findings about constraint violations and $\mathrm{HC}$ values, when more than one constraints are violating and margin among them for the decision of primary violated constraint is very small.

However, for the third stage of the algorithm, the small number of iterations are utilized as the number of worst-case hours found are small (right part of Figures 2 and 3). If the large number of iterations are done, the result will be based on the recurring values employed for the MC simulation of HC determination. Hence, the large number of third stage iterations leads to excessive computations, without affecting the final result. 


\subsection{Grid Topologies Comparison}

The grid topologies used are different based on the MV change scenarios (number of nodes are different), as defined in Table 1. Loading nodes will have an effect on the HC value of a particular region scenario and MV changes $(0 \%,+5 \%$ and $-5 \%)$. The MV voltage level propagates downstream to customers and affecting its voltage levels. However, the customer acceptable voltage levels remain the same. For instance, the $+5 \%$ MV voltage changes leave less margin for overvoltage as the HC is limited by the upper voltage limit. The $-5 \%$ voltage change leaves more headroom for voltage rise.

Moreover, the grid topologies of the PR region utilized are weak grids, which accounts for the drastic HC changes. Small introduction of single phase PV will greatly reduce the HC and change the limiting constraint to E4. In contrast, in the IN region, the single-phase PV inclusion is greater than the $\mathrm{PR}$ region for all the MV change scenarios compared with the PR region.

\subsection{Cases Comparison}

HCs for all the MV change values for different cases and regions (PR, IN) are presented by the bar graphs of Figures 5 and 6. In PR region, when case A is implemented, E4 (voltage unbalance) is limiting the $\mathrm{HC}$ even at $5 \%$ of the single-phase PV connections for all the MV change scenarios. BESS random connection among the phases increases the voltage unbalance and the $\mathrm{HCs}^{\prime}$ are close to $20 \%$ for all the simulated scenarios. Nevertheless, in case B, as the battery is attached to the same phase as the PV, the HCs are drastically increased. The single-phase PV percentage rises to $60 \%, 20 \%$ and $100 \%$ for $0 \%$, $-5 \%$ and $+5 \%$ MV change cases, respectively. The largest increment in $\mathrm{HC}$ is seen in the $+5 \%$ case, in which the HC is quadrupled. In case C, the load unbalance is also incorporated, which in theory should reduce the HCs for the E4-dependent scenarios. The single-phase PV connections reduction in $0 \% \mathrm{MV}$ change case is $15 \%$ and $5 \%$ for the $\pm 5 \% \mathrm{MV}$ change scenarios. With the increased voltage unbalance, E4 is violated earlier than in case B, lesser unbalanced PVs will lead to the increased HCs. Nevertheless, in $+5 \%$ MV change scenario, $\mathrm{HC}$ is decreased irrespective of the reduced percentage of unbalanced PVs. This signifies that the unbalance increment, due to the load unbalance addition, is larger than the unbalance reduction due to $5 \%$ increment in the balanced PVs.

For the IN region, the similar trend as the PR region is observed. For case A, 5\% unbalanced $\mathrm{PV}$ connection in the network are suffice for E4 violations. Moreover, the case B implementation increases the unbalanced PV percentages to $80 \%, 65 \%$ and $90 \%$ for $0 \%,-5 \%$ and $+5 \%$ MV change cases, respectively. Lastly, for the case $\mathrm{C}, \mathrm{HCs}$ are slightly reduced due to load unbalance incorporation.

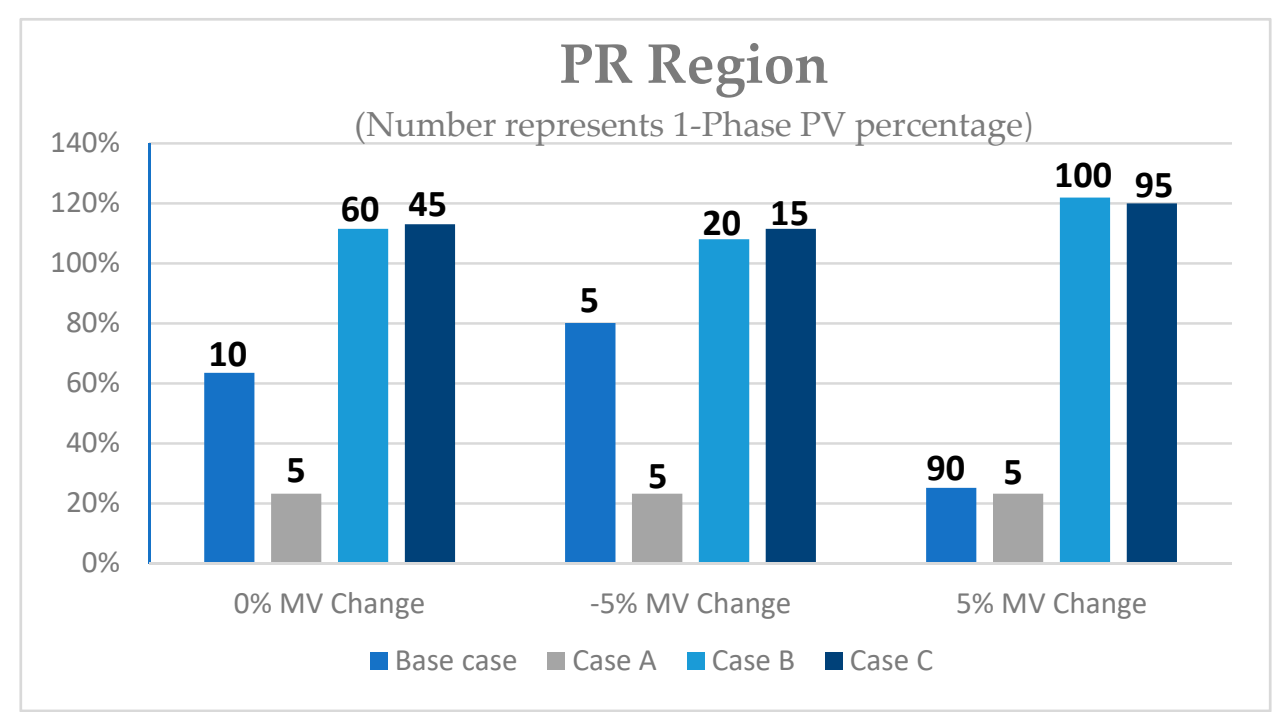

Figure 5. PV hosting capacities for various cases in PR region (E4 as the limiting constraint). 


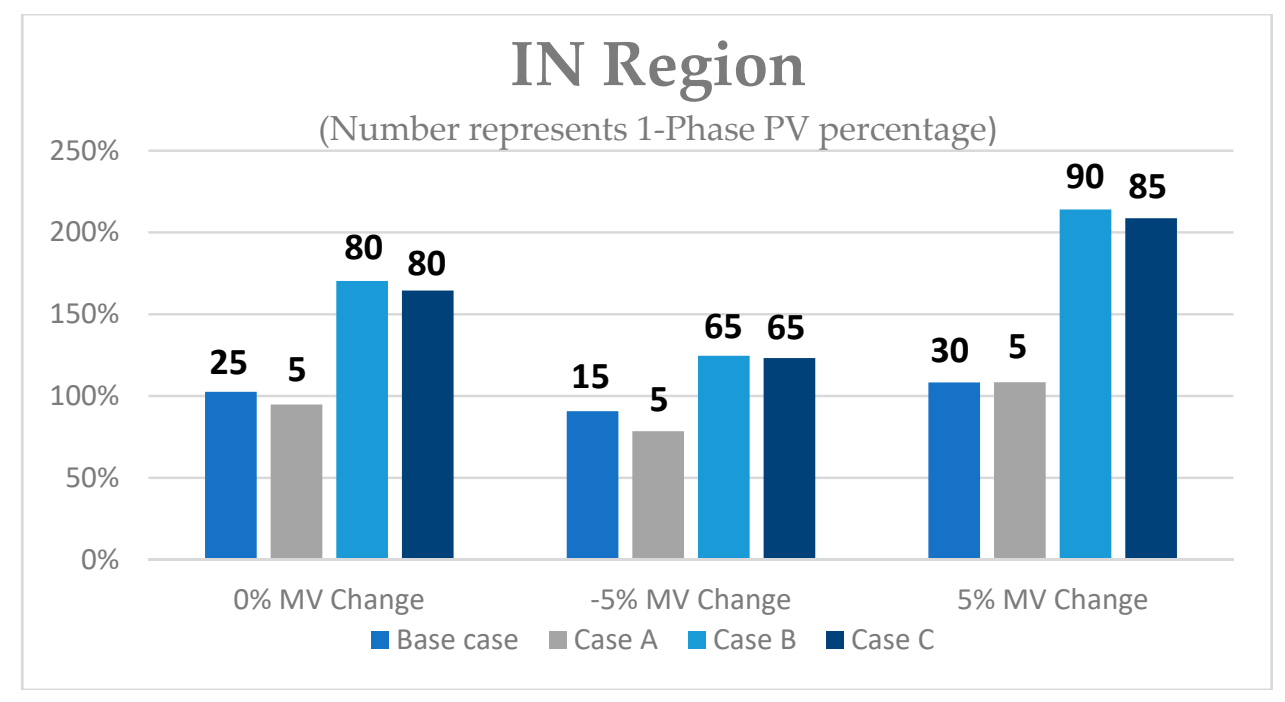

Figure 6. PV hosting capacities for various cases in IN region (E4 as the limiting constraint).

\section{Conclusions}

A Monte-Carlo-based study was conducted to quantify the effect of different voltage standards and BESS employment to the HC of the LV networks. Moreover, the percentage of unbalanced PV inclusion in the network was assessed for different regions and varying scenarios.

Firstly, the HCs of the various region-dependent networks and their limiting constraints were assessed for the standard EN 50160. Afterwards, it was compared with the different country-dependent voltage standards. The result indicates that, for balanced PVs, as the voltage limit was made stricter, the constraint limiting the HC in PR region was shifting towards E3 (Overvoltage) and HCs were also drastically decreased. However, for IN and PU region E7 (Transformer capacity) remains the limiting factor for networks' HCs. However, for unbalanced PV cases, all the HCs and limiting constraints remain the same for different voltage band limits i.e., E4 (voltage unbalance), except for the German LV standard where the limit change to E3 and HC was also slightly decreased.

Secondly, unbalanced PV percentage inclusion was computed, and different BESS connections were assessed for their impact on the HCs and voltage unbalance reduction. As for single-phase percentage inclusion, in PR region, $-5 \%$ and $0 \% \mathrm{MV}$ change scenarios can have up to $10 \%$ unbalanced PV connections. However, in $+5 \%$ MV change case, up to $90 \%$ of single PV connections can be tolerated without voltage unbalance as the limiting factor of HC. As for the IN region, for all the MV cases, the limit of unbalanced PV is less than $30 \%$.

Lastly, BESS cases were studied, and the results signify that if the BESS was randomly distributed among the phases, unbalance increases, and HCs were greatly reduced for PR region. However, in the IN region the decrement in HCs is not very abrupt. Moreover, single-phase PV connection percentage is also restricted to $5 \%$ of the total PV inclusion for all the simulated cases dependent on regions and MV changes. However, as the BESS is employed on the same phase as the single-phase PV, the HCs mount to a rather greater value than the case that employs PV-only strategy. Moreover, load unbalance is also incorporated in the last case that signifies that PR region was more inclined to show changes in the single-phase PV percentage reduction with the increased unbalanced introduced by the load unbalance. Nevertheless, in IN region, the percentage remains the same, yet the HCs were decreased slightly.

Author Contributions: A.A. developed the framework for hosting capacity assessment, performed simulations and wrote the paper. V.P. introduced the battery model, processed the load voltage-unbalance data and contributed in the final draft of the paper. M.L. supervised the work and commented on paper structure, language and format.

Acknowledgments: This work has been supported by department of Electrical Engineering and Automation of Aalto University, Finland and the Finnish Solar Revolution project of Business Finland. 
Conflicts of Interest: The authors declare no conflict of interest.

\section{Appendix A}

Table A1. Loading distribution based on heating modes.

\begin{tabular}{cccc}
\hline \multirow{2}{*}{ Regions } & \multicolumn{3}{c}{ Heating Modes } \\
\cline { 2 - 4 } & $\begin{array}{c}\text { Storage Heating } \\
\text { (SH) (\%) }\end{array}$ & $\begin{array}{c}\text { Non-Electric Heating } \\
\text { (NEH) (\%) }\end{array}$ & $\begin{array}{c}\text { Direct Electric Heating } \\
\text { (DEH) (\%) }\end{array}$ \\
\hline Predominantly Rural (PR) & 5.9 & 52.9 & 41.2 \\
Intermediate (IN) & 7.6 & 52.5 & 39.9 \\
Predominantly Urban (PU) & 0.4 & 95.3 & 4.2 \\
\hline
\end{tabular}

Table A2. LV Network parameters for different regions.

\begin{tabular}{ccccc}
\hline Region & Transformer $\boldsymbol{S}_{\boldsymbol{r}}(\boldsymbol{k V \boldsymbol { A } )}$ & Cable Length $(\mathbf{m})$ & Customer per Node & Cable \\
\hline PR & 50 & 150 & 1 & $A X M K 4 \times 70 \mathrm{~mm}^{2}$ \\
IN & 200 & 100 & 4 & $A X M K 4 \times 185 \mathrm{~mm}^{2}$ \\
PU & 1000 & 100 & 60 & $2 \times A X M K 4 \times 185 \mathrm{~mm}^{2}$ \\
\hline
\end{tabular}

\section{References}

1. Walling, R.; Saint, R.; Dugan, R.C.; Burke, J.; Kojovic, L.A. Summary of distributed resources impact on power delivery systems. IEEE Trans. Power Del. 2008, 23, 1636-1644. [CrossRef]

2. Shahnia, F.; Majumder, R.; Ghosh, A.; Ledwich, G.; Zare, F. Sensitivity analysis of voltage imbalance in distribution networks with rooftop PVs. In Proceedings of the Power and Energy Society General Meeting, Providence, RI, USA, 25-29 July 2010; pp. 1-8.

3. Seuss, J.; Reno, M.J.; Broderick, R.J.; Grijalva, S. Improving distribution network PV hosting capacity via smart inverter reactive power support. In Proceedings of the Power \& Energy Society General Meeting, Denver, CO, USA, 26-30 July 2015; pp. 1-5.

4. Stetz, T.; Marten, F.; Braun, M. Improved low voltage grid-integration of photovoltaic systems in Germany. IEEE Trans. Sustain. Energy 2013, 4, 534-542. [CrossRef]

5. Bletterie, B.; Gorsek, A.; Uljanic, B.; Blazic, B.; Woyte, A.; Van, T.V.; Truyens, F.; Jahn, J. Enhancement of the network hosting capacity-Clearing space for/with PV. In Proceedings of the 25th European Photovoltaic Solar Energy Conference and Exhibition, Valencia, Spain, 6-10 September 2010; pp. 4828-4834.

6. Rylander, M.; Smith, J.; Sunderman, W. Streamlined method for determining distribution system hosting capacity. IEEE Trans. Ind. Appl. 2016, 52, 105-111. [CrossRef]

7. Shayani, R.A.; Oliveira, D.; Gonçalves, M.A. Photovoltaic generation penetration limits in radial distribution systems. IEEE Trans. Power Syst. 2011, 26, 1625-1631. [CrossRef]

8. Kordkheili, R.A.; Bak-Jensen, B.; R-Pillai, J.; Mahat, P. Determining maximum photovoltaic penetration in a distribution grid considering grid operation limits. In Proceedings of the PES General Meeting I Conference \& Exposition, National Harbor, MD, USA, 27-31 July 2014; pp. 1-5.

9. Ding, F.; Mather, B.; Gotseff, P. Technologies to increase PV hosting capacity in distribution feeders. In Proceedings of the Power and Energy Society General Meeting (PESGM), Boston, MA, USA, 17-21 July 2016; pp. 1-5.

10. Patsalides, M.; Makrides, G.; Stavrou, A.; Efthymiou, V.; Georghiou, G.E. Assessing the photovoltaic (PV) hosting capacity of distribution grids. In Proceedings of the MedPower Conference Proceedings, Belgrade, Serbia, 6-9 November 2016.

11. Arshad, A.; Lindner, M.; Lehtonen, M. An Analysis of Photo-Voltaic Hosting Capacity in Finnish Low Voltage Distribution Networks. Energies 2017, 10, 1702. [CrossRef]

12. Schwanz, D.; Möller, F.; Rönnberg, S.K.; Jan, M.; Bollen, M.H.J. Stochastic assessment of voltage unbalance due to single-phase-connected solar power. IEEE Trans. Power Del. 2017, 32, 852-861. [CrossRef] 
13. Chua, K.H.; Wong, J.; Lim, Y.S.; Taylor, P.; Morris, E.; Morris, S. Mitigation of voltage unbalance in low voltage distribution network with high level of photovoltaic system. Energy Procedia 2011, 12, 495-501. [CrossRef]

14. Chua, K.; Lim, Y.S.; Taylor, P.; Morris, S.; Wong, J. Energy storage system for mitigating voltage unbalance on low-voltage networks with photovoltaic systems. IEEE Trans. Power Del. 2012, 27, 1783-1790. [CrossRef]

15. Wong, J.; Lim, Y.S.; Morris, S.; Morris, E.; Chua, K.H. Fuzzy-driven energy storage system for mitigating voltage unbalance factor on distribution network with photovoltaic system. AIP Conf. Proc. 2017, 020019. [CrossRef]

16. Čumpelík, R.; Krejčí, P. Undefined parameters and two-limit parameters in standard EN 50160 ED. 3. In Proceedings of the 2015 16th International Scientific Conference on Electric Power Engineering (EPE), Kouty nad Desnou, Czech Republic, 20-22 May 2015; pp. 433-436.

17. Viotto, M. Present Status of DG in Germany: National Codes, Standards, Requirements and Rules for Grid-Interconnection and Operation; Technical report; ISET: Kassel, Germany, April 2005.

18. Ekström, J.; Koivisto, M.; Mellin, I.; Millar, R.J.; Lehtonen, M. A statistical approach for hourly photovoltaic power generation modeling with generation locations without measured data. Solar Energy 2016, 132, 173-187. [CrossRef]

19. TESLA INC. Tesla Power Wall 2 AC Datasheet. 2016. Available online: https://www.energymatters.com. au/wp-content/uploads/2016/11/tesla-powerwall-2-datasheet.pdf (accessed on 25 February 2018).

(C) 2018 by the authors. Licensee MDPI, Basel, Switzerland. This article is an open access article distributed under the terms and conditions of the Creative Commons Attribution (CC BY) license (http://creativecommons.org/licenses/by/4.0/). 